\title{
NLS1 properties and demographics (invited)
}

\section{Todd Boroson*}

National Optical Astronomy Observatory ${ }^{\dagger}$

E-mail: tybenoao.edu

Archives of tens or even hundreds of thousands of active galaxy spectra allow us to probe the demographics of AGNs, and in particular, NLS1s, in new ways and to study relationships among properties that would not be visible in smaller samples. Using the Sloan Digital Sky Survey database and published automated measurements of spectral features, it is possible to determine whether the properties that are associated with NLS1s are really different in these objects. Ultimately, the goal is to understand the nature of NLS1s in the context of the basic physical parameters that govern the observed properties of AGNs.

In this contribution I use measurements of line and continuum properties in SDSS spectra of thousands of low-redshift QSOs to show how these observed characteristics are related to broad line width, the fundamental discriminator of the objects called NLS1s. Exploration of these correlations and of properties derived from the observables allows the separation of the effects of black hole mass, Eddington ratio, and orientation. I find that NLS1s are the class of AGN in the local universe that have the smallest black holes and the largest Eddington ratios. I also find that they are seen preferentially pole-on, and while this contributes to their extreme properties, it is not the dominant driver.

Narrow-Line Seyfert 1 Galaxies and their place in the Universe

April 4-6, 2011

Milano, Italy

\footnotetext{
* Speaker.

$\dagger$ The National Optical Astronomy Observatory is operated by AURA, Inc. under cooperative agreement with the National Science Foundation.
} 


\section{Introduction}

The pioneering work of Osterbrock and Pogge [8] identified a class of Seyfert 1 galaxies with exceptionally narrow Balmer lines. They obtained spectra of a small set of Seyfert galaxies that were known to have $\mathrm{H} \beta$ emission line full width at half-maximum $\left(\mathrm{FWHM}_{\mathrm{H} \beta}\right)$ only slightly broader than the forbidden lines, typically a few hundred $\mathrm{km} \mathrm{s}^{-1}$. These authors found that other properties, forbidden line widths, relative line strengths, in these objects are all indicative of Seyfert 1 galaxies. They also noted that other than their $\mathrm{H} \beta$ equivalent widths $\left(\mathrm{EW}_{\mathrm{H} \beta}\right)$ being at the small end of the Seyfert 1 distribution, other properties showed diversity within typical ranges.

As samples of AGN spectra grew in size, it was recognized that these narrow-line Seyfert 1 galaxies (NLS1) are a significant subset of the AGN population. Using the dividing line of $\mathrm{FWHM}_{\mathrm{H} \beta}=2000 \mathrm{~km} \mathrm{~s}^{-1}$, NLS1s make up about $15 \%$ of all broad line AGN [20]. Studies of their properties have considered larger and larger numbers of objects [15, 20]. A good recent review of their properties is given by Komossa [6].

Beyond the properties that define the class, $\mathrm{FWHM}_{\mathrm{H} \beta}<2000 \mathrm{~km} \mathrm{~s}^{-1}$ and $[\mathrm{O} \mathrm{III}] \lambda 5007 / \mathrm{H} \beta_{\text {total }}$ $<3$, NLS1s typically show strong optical Fe II emission and a tendency for blueshifts or blue wings on the high-ionization emission lines, both narrow (e.g., [O III]) [19, 1] and broad (e.g., C IV)[14, 17]. They are less often very radio-loud and tend to have redder continuum slopes in the optical region. Their x-ray spectra show more diversity in slope than AGNs with broader permitted lines. Many of these trends are members of the set of correlations known as Eigenvector 1 (EV1) [2], and consequently, NLS1s are found at one end of the EV1 sequence.

The EV1 set of correlations is interesting both because it represents the largest fraction of variance in the optical spectra of AGN and because it seem to link properties that are indicative of processes and conditions in a variety of regions. Thus, some fundamental physical driver must account for it, and NLS1s represent the extreme of whatever that driver is. While much evidence points to $\mathrm{L} / \mathrm{L}_{\mathrm{Edd}}$ (Eddington ratio) as the primary driver, other factors may contribute. For instance, it seems likely that the broad line region (BLR) and continuum emitting region are not spherical but disklike, and so it might be expected that there are orientation effects that influence line widths and strengths[3, \#. In addition, there is the question of where NLS1s lie in the $\mathrm{M}_{\mathrm{BH}}-\sigma_{\star}$ diagram. Some studies have found that they lie on or close to the line defined by non-active galaxies and AGN with broader lines[7], but some studies have found that NLS1s have smaller black hole masses at a given velocity dispersion[20]. This is interpreted as an indication that they are younger than broader line AGN and are in the process of increasing their black hole masses by accretion.

The Sloan Digital Sky Survey (SDSS) [18] is a uniquely powerful tool for understanding the properties of various classes of AGN. Good quality spectra and photometric properties of more than 100,000 AGN allow detailed and significant analyses of how properties are related. Subsets can be chosen from the full sample to mimic controlled experiments. The SDSS QSO catalog [11] has a lower luminosity limit of $\mathrm{M}_{i}<-22$, and so in this study, I interpret NLS1 to mean any Type 1 AGN having FWHM $_{\mathrm{H} \beta}<2000 \mathrm{~km} \mathrm{~s}^{-1}$, regardless of luminosity. In fact, my conclusions will be drawn primarily from data on objects that would be considered QSOs rather than Seyfert galaxies.

In this contribution, I use the SDSS archive of QSO spectra to explore how some simple, observed properties vary with broad line width. This leads to the finding that NLS1s are the AGN in the local universe that have, on average, the smallest black hole masses and the largest Eddington 
ratios. Then, I introduce measurements of systematic velocity shifts of broad and narrow lines in a diagram that allows the separation of orientation effects from physical parameters. The orientation effects, and their impact on observed properties can be quantified. Ultimately, I find that it is a combination of all of these drivers that produce the properties of NLS1s.

\section{Properties that vary with FWHM of $\mathrm{H} \beta$}

Although many studies have measured properties of NLS1s and compared their distributions to those of broader line AGN[15, 20], the SDSS data set allows a much more complete picture. In order to put statistical measurements in the proper context, I start by reviewing the sample constraints for the SDSS spectra in general, for the SDSS DR7 QSO catalog[11], and for 3 studies that published measured properties for SDSS objects, which I will use to derive correlations.

The SDSS (the Sloan Legacy Survey) [18] carried out 5-color imaging over about 8400 square degrees, and used the resulting photometric information to select about one million galaxies and 100,000 QSOs for spectroscopy. The selection criteria produced three fairly complete samples, galaxies, luminous red galaxies, and QSOs, though a significant number of spectra were obtained of objects that do not fall into these samples. The galaxy sample includes objects that are resolved spatially and are brighter than a threshold of about $r=18$. The luminous red galaxy sample is aimed at identifying red galaxies out to a larger redshift than the main galaxy sample. It is defined using a number of color and magnitude cuts in the $\mathrm{g}, \mathrm{r}$, and $\mathrm{i}$ bands. The QSO sample is defined without regard to whether objects are stellar or extended. It includes a number of color cuts designed to find the objects that have colors outside of the stellar locus. There is also a magnitude limit, $\mathrm{i}<$ 19.1, but fainter objects are included if they have other properties consistent with identification as high redshift QSOs. A large fraction of the objects targeted in these samples were observed spectroscopically, and the SDSS spectra cover the observed wavelength range $\lambda \lambda$ 3800-9200.

The SDSS DR7 QSO catalog includes the 105,783 objects observed spectroscopically in the Sloan Legacy Survey that are officially confirmed as QSOs. Two additional restrictions are applied, one for luminosity and one for broad line width. To be classified as a QSO in this catalog, objects must have a luminosity in the de-redshifted i band brighter than $\mathrm{M}_{i}=22$, and they must have a broad line width of more than $1000 \mathrm{~km} \mathrm{~s}^{-1}$ FWHM. Both of these restrictions exclude some objects that would be classified as NLS1s.

There are three published large tables of measurements of line and continuum properties from the SDSS spectra that I will use in this study. The S10 study [12] presents a table of properties, measured by an automated decomposition, of all the objects in the SDSS DR7 QSO catalog. This table includes broad and narrow line widths, fluxes and velocities, and continuum fluxes and slopes. Fe II emission strengths are measured by fitting to empirical models. The broad line model is the sum of two gaussians, one with properties consistent with the narrow, forbidden lines. In the current study, I use a subset of the full sample, limited to objects from the uniform samples with $\mathrm{z}<0.8$ and $\mathrm{S} / \mathrm{N}>5$. These cuts result in 10,240 objects, of which 426 would be classified NLS1s.

The H08 study [5] presents a table of line and continuum properties for a subset of QSOs from the SDSS DR5 QSO [10] catalog. This study is aimed at understanding the behavior of the optical Fe II emission, and so the sample is limited to 4037 objects that have good signal-to-noise $(\mathrm{S} / \mathrm{N}>10)$ and significant $\mathrm{Fe}$ II $\left(\mathrm{EW}_{\mathrm{FeII}}>25 \AA\right)$. This work reports a number of tests of the accuracy 
of the continuum decomposition, and, in particular, it is shown that the Fe II measurements are robust.

The Z06 study [20] explored the properties of NLS1s specifically and tabulates emission line and continuum properties for about 2000 objects having $\mathrm{FWHM}_{\mathrm{H} \beta}<2200 \mathrm{~km} \mathrm{~s}^{-1}$ drawn from the SDSS DR3 archive. This sample is not limited to SDSS QSOs, but includes objects from the galaxy samples that were too low in luminosity or broad line width to be included in the spectroscopic QSO sample. Because such low luminosity nuclei are included, significant starlight contributions were removed from some of the objects in their sample.

One can, for example, use these samples to address the question of whether NLS1s represent a distinct population or are just the extremes of a set of properties that vary smoothly over AGN generally. The S10 sample was sorted by $\mathrm{FWHM}_{\mathrm{H} \beta}$ and divided into 20 subsets with equal numbers of objects $(\sim 500)$ in each subset. Figure 1 shows the behavior of four properties plotted against $\mathrm{FWHM}_{\mathrm{H} \beta}: \mathrm{EW}_{[\mathrm{OIII}]}, \mathrm{EW}_{\mathrm{FeII}}, \mathrm{EW}_{\mathrm{H} \beta}$ and $\log \mathrm{L}_{5100}$. For each subset, median values of both the EWs (or L) and the $\mathrm{FWHM}_{\mathrm{H} \beta}$ are shown. Interestingly, $\mathrm{EW}_{[\mathrm{OIII}]}$ and $\mathrm{EW}_{\mathrm{FeII}}$ appear to vary continuously over the entire range of $\mathrm{FWHM}_{\mathrm{H} \beta}$ while $\mathrm{EW}_{\mathrm{H} \beta}$ and $\log \mathrm{L}_{5100}$ appear to change their behavior in a dramatic way at the threshold of NLS1s. The trends shown by the panels on the left are extremely weak in the full sample, in the sense that the scatter within any subset is much larger than the difference between subsets. For example, the individual object correlation coefficients for the two panels on the left are $\mathrm{r}=0.08$ for the relation between $\mathrm{EW}_{[\mathrm{OIII}]}$ and $\mathrm{FWHM}_{\mathrm{H} \beta}$ and $\mathrm{r}=0.01$ for the relation between $\mathrm{EW}_{\mathrm{FeII}}$ and $\mathrm{FWHM}_{\mathrm{H} \beta}$, though it is clear from the plot of the medians that the correlations are quite real.

Similarly, the panels on the right show that, when viewed only as a function of $\mathrm{FWHM}_{\mathrm{H} \beta}$, the median luminosity changes behavior around $3000 \mathrm{~km} \mathrm{~s}^{-1}$ and drops below that value, and that the luminosity of the $\mathrm{H} \beta$ line drops even more quickly, causing $\mathrm{EW}_{\mathrm{H} \beta}$ to decrease correspondingly. These variations, too, are quite significant, though one would never see them in the individual objects or with a smaller sample.

\section{Black Hole Mass and Eddington Ratio}

One of the fundamental questions about NLS1s is whether they represent a set of AGNs that has small back hole mass $\left(\mathrm{M}_{\mathrm{BH}}\right)$ and/or is accreting close to their Eddington limit. It is worth pointing out that the Eddington limit is often applied to arguments about AGNs, but it is not at all clear that it applies. First, the ratio between mass and luminosity $\left(\mathrm{L}_{\mathrm{Edd}} / \mathrm{L}_{\odot}=3.3 \times 10^{4} \mathrm{M} / \mathrm{M}_{\odot}\right)$ applies to spherical accretion and isotropic emission of radiation, which is likely not the case in AGN. Second, the limit represents the balance of radiation pressure through Thompson scattering against gravity. Once the material involved, broad line clouds, for example, become optically thick, the interior atoms will not see the radiation, though they will feel the gravity.

The standard formalism for calculating $\mathrm{M}_{\mathrm{BH}}$ is to assume virial equilibrium, and to use a measure of the broad line width, in this case $\mathrm{FWHM}_{\mathrm{H} \beta}$, and a measure of the continuum luminosity, in this case $\mathrm{L}_{5100}$, as indicators of the BLR velocity dispersion and the bolometric luminosity of the AGN. The bolometric luminosity has been found to be correlated with the size of the BLR, and so the black hole mass can be calculated, to within a constant that depends on the anisotropy in the BLR velocity distribution. Two caveats should be mentioned. First, reverberation mapping 

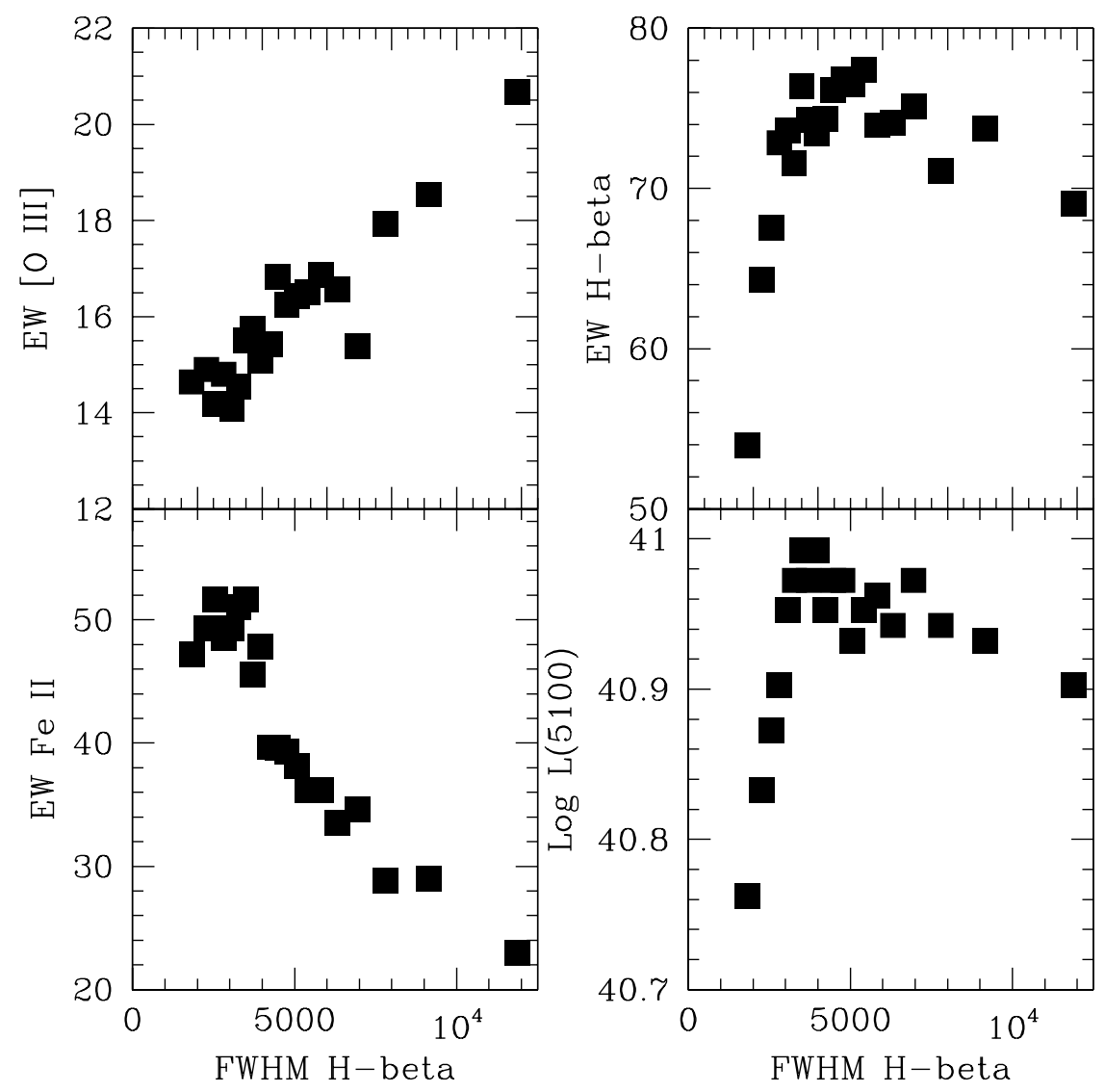

Figure 1: Four examples of extremely significant correlations seen by sorting a large sample by $\mathrm{FWHM}_{\mathrm{H} \beta}$, defining 20 subsamples, and plotting the median value of (top left) equivalent width of [O III] $\lambda 5007$, (bottom left) equivalent width of Fe II $\lambda \lambda$ 4434-4684, (top right) equivalent width of $\mathrm{H} \beta$, and (bottom right) $\log$ of the monochromatic luminosity at $\lambda 5100$ against the median value of $\mathrm{FWHM}_{\mathrm{H} \beta}$ for each subsample.

measurements of the size of the BLR involve determining a lag in response to continuum changes by material at a "typical" radius corresponding to the peak of the cross-correlation. Since we typically measure single-epoch spectra, there is an assumption that the line width we measure is related to material at a radius that has some simple relationship to that cross-correlation radius. Second, while FWHM is an easy quantity to measure, particularly if the line is fit to some simple function like a Gaussian, the relationship between this quantity and the velocity dispersion is a function of the line shape. Since broad lines show a trend between line width and line shape, there may be systematic errors of factors of several between objects having relatively narrow and relatively broad lines.

With these caveats in mind, we can calculate $\mathrm{M}_{\mathrm{BH}}$ and Eddington ratio for each object in the S10 sample. We use the equations [16]:

$$
\begin{gathered}
\log \mathrm{M}_{\mathrm{BH}}=0.91+0.5\left(\log \lambda \mathrm{L}_{5100}-44.0\right)+2 \log \mathrm{FWHM}_{\mathrm{H} \beta} \\
\log \mathrm{L} / \mathrm{L}_{\mathrm{Edd}}=\log \left(9.26 \lambda \mathrm{L}_{5100}\right)-\log \mathrm{M}_{\mathrm{BH}}-38.10
\end{gathered}
$$




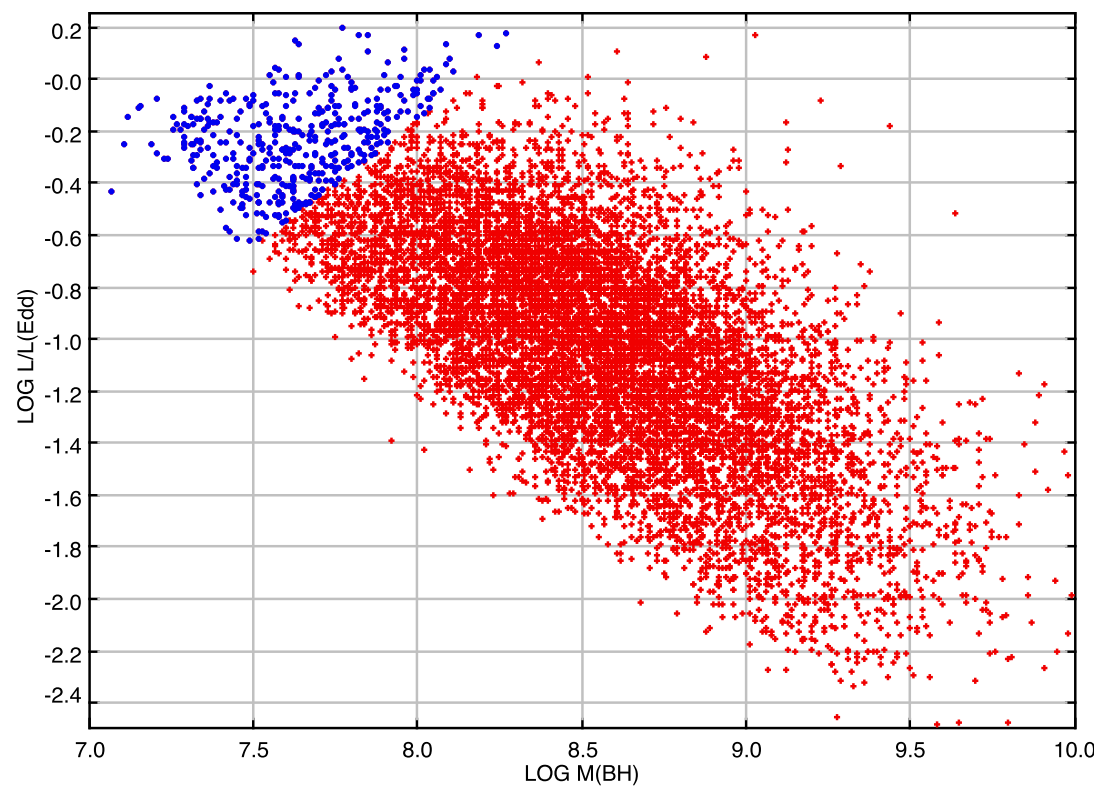

Figure 2: The locus of 10,240 low redshift AGN from the study of Shen et al. (2010) in the log Eddington ratio vs. $\log \mathrm{M}_{\mathrm{BH}}$ plane. The NLS1s, having $\mathrm{FWHM}_{\mathrm{H} \beta}<2000 \mathrm{~km} \mathrm{~s}^{-1}$, are shown in blue.

Figure 2 plots $\log \mathrm{L} / \mathrm{L}_{\mathrm{Edd}}$ against $\log \mathrm{M}_{\mathrm{BH}}$ with the NLS1s shown in blue. The lower luminosity limit of the SDSS QSO catalog is responsible for the sharp cutoff towards the lower left corner. However, the lack of objects in the upper right corner is a significant result. It appears that in the local universe $(\mathrm{z}<0.8)$, NLS1s are emitting at close to their Eddington limit and are the only AGNs doing so. This is apparently the same effect as the recently proposed "Sub-Eddington Boundary" (SEB) [13].

There are three reasons to question this result. First, we are effectively plotting L/M against $\mathrm{M}$, so any errors in $\mathrm{M}$ will stretch the distribution of points out in the diagonal direction that we see here. Second, it is possible that the systematic trend in line shape with line width could produce this effect - small line width objects have profiles that result in relatively smaller FWHM values, and this reduces their apparent $\mathrm{M}_{\mathrm{BH}}$ and increases their apparent Eddington Ratio. Third, the SDSS QSO luminosity threshold comes uncomfortably close to the upper limit in Eddington Ratio at the NLS1 end of the distribution. Perhaps there is a large number of excluded low $\mathrm{M}_{\mathrm{BH}}$ objects with lower Eddington Ratio that were excluded, and the true median of the distribution at the left end is far lower in the diagram.

The first concern can be addressed by recognizing that $\log \mathrm{M}_{\mathrm{BH}}$ and $\log$ Eddington Ratio are linear combinations of $\log \mathrm{L}_{5100}$ and $\log \mathrm{FWHM}_{\mathrm{H} \beta}$. Thus, we can examine the same effect by plotting these two observed quantities (Figure 3 ) for which the errors are uncorrelated. With the exponents adopted above in the $\mathrm{M}_{\mathrm{BH}}$ equation, the slope of a line of constant Eddington Ratio is 4. As is clear in Figure 3 , this line passes two orders of magnitude above the points at $\mathrm{FWHM}_{\mathrm{H} \beta}$. How would the exponents have to change to make the line consistent with the points? Only if $\mathrm{M}_{\mathrm{BH}}$ 


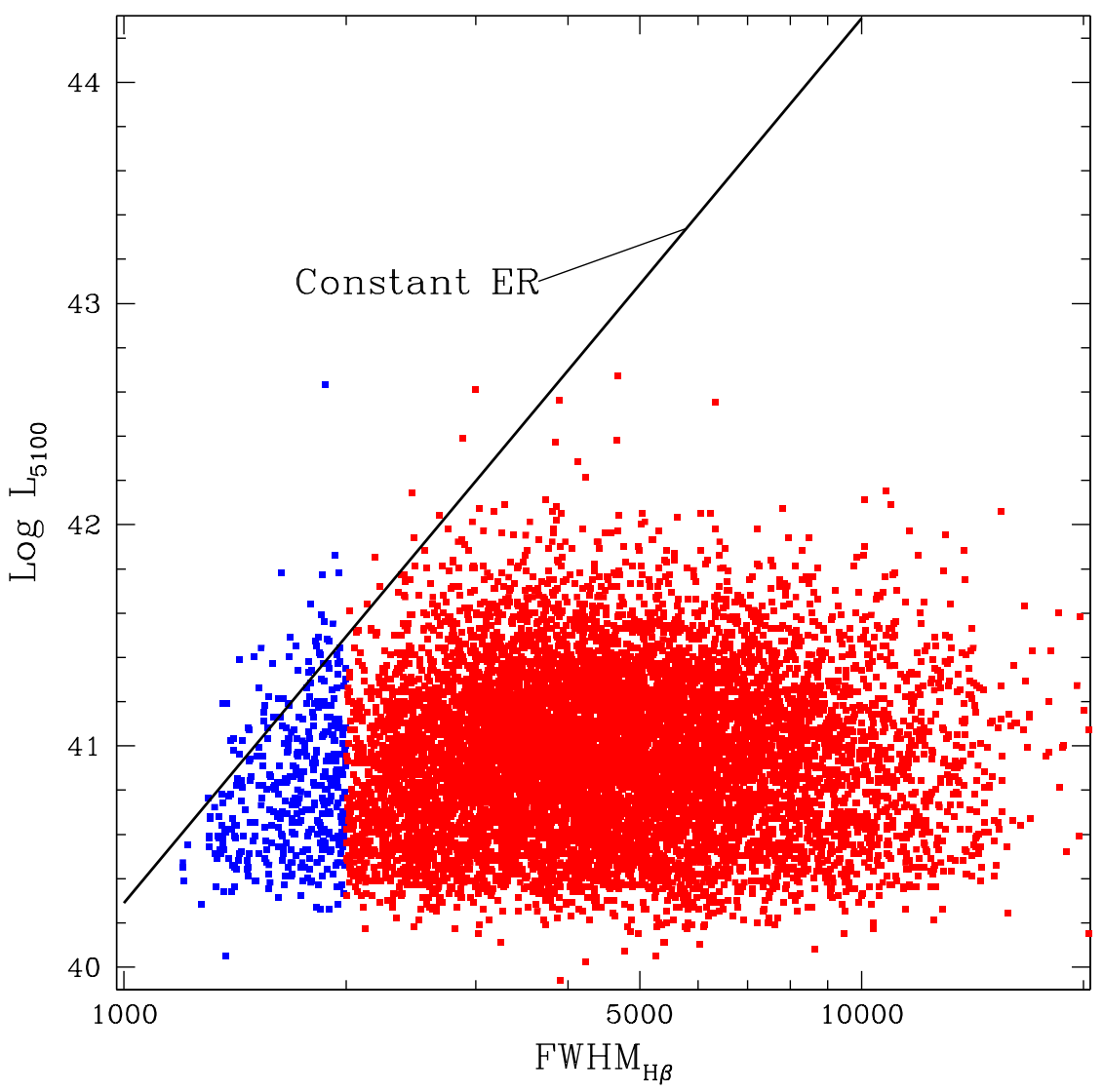

Figure 3: The locus of 10,240 low redshift AGN from the study of Shen et al. (2010) in the $\log \mathrm{L}_{5} 100$ vs. $\log \mathrm{FWHM}_{\mathrm{H} \beta}$ plane. The NLS1s, having $\mathrm{FWHM}_{\mathrm{H} \beta}<2000 \mathrm{~km} \mathrm{~s}^{-1}$, are shown in blue. A line of constant Eddington ratio, using the conventional parameters, is shown.

were almost independent of $\mathrm{FWHM}_{\mathrm{H} \beta}$ would this effect be eliminated.

The second concern, that we are being misled by a systematic trend in line shape with line width, can be addressed by using the $\mathrm{Hu}$ et al. [5] dataset. These authors measure and use $\sigma_{\mathrm{H} \beta}$ rather than $\mathrm{FWHM}_{\mathrm{H} \beta}$ in their calculation of $\mathrm{M}_{\mathrm{BH}}$. The locus of points for these 4037 objects is shown in Figure 月, with the NLS1s again shown in blue. It can be seen that the upper right corner where objects with both large $\mathrm{M}_{\mathrm{BH}}$ and large Eddington Ratio would be found is still relatively empty. Thus, this effect cannot explain the SEB.

Finally, we address the possibility that it is the lower luminosity limit of the SDSS QSO catalog that is creating the impression of an excess of objects with high Eddington Ratio and low $\mathrm{M}_{\mathrm{BH}}$. To do this, we use the Z06 [20] sample, which is limited to NLS1s, but is drawn from the full SDSS spectroscopic database rather than from the SDSS QSO catalog. The mean value of $\mathrm{L} / \mathrm{L}_{\mathrm{Edd}}$ for this sample is -0.47 . This is a little below the mean value of $\mathrm{L} / \mathrm{L}_{\text {Edd }}$ for the NLS1s in the S10 sample, but it is still well above the mean value of -1.50 for all the S10 AGN with $\log \mathrm{M}_{\mathrm{BH}}$ greater than 9 . Thus, while the true distribution of AGN in the $\log$ Eddington Ratio vs. $\log \mathrm{M}_{\mathrm{BH}}$ plane may not be as steep as it appears in Figure 2, it is still substantially tilted. 


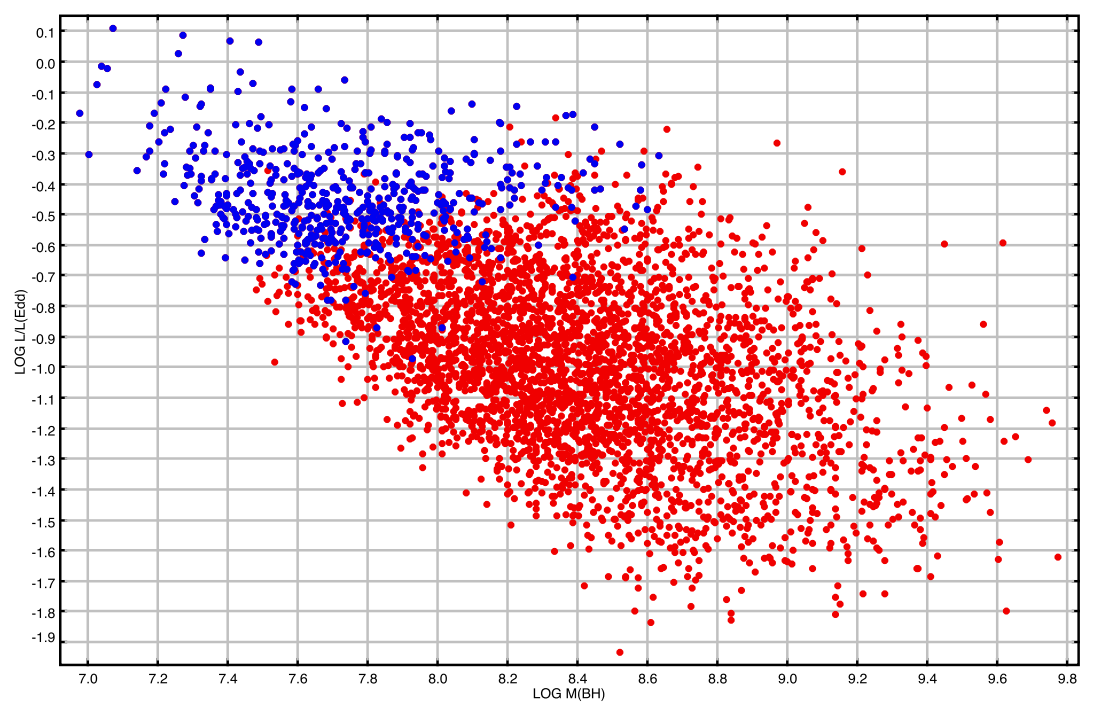

Figure 4: The locus of 4,037 low redshift AGN from the study of Hu et al. (2008) in the log Eddington ratio vs. $\log \mathrm{M}_{\mathrm{BH}}$ plane. In this study, the $\mathrm{M}_{\mathrm{BH}}$ values are calculated using $\sigma_{\mathrm{H} \beta}$ rather than $\mathrm{FWHM}_{\mathrm{H} \beta}$. The NLS1s, having FWHM $_{\mathrm{H} \beta}<2000 \mathrm{~km} \mathrm{~s}^{-1}$, are shown in blue.

From this analysis, it is clear that NLS1s are the AGN with, on average, the smallest black hole masses and the largest Eddington Ratios.

\section{New Evidence that Orientation is Important}

Despite the evidence that NLS1s show high Eddington Ratios and small black hole masses, there remains the possibility that some of their properties are due, at least in part, to a preferred orientation. It has long been recognized that AGNs are not spherical. In particular, the optical continuum and the low ionization broad emission lines show evidence of coming from a flattened distribution of material, which may be related to the accretion disk. The narrow lines are believed to come from a biconical region, perpendicular to that disk. The consequence of such a geometry would be that pole-on objects would appear to be more luminous and have narrower broad lines than edge-on objects. One recent study that lends support to this picture examines the distribution of [O III] $\lambda 5007$ equivalent width in the S10 sample[9]. This study compares that distribution with a simple model in which the line emission is isotropic and the continuum emission comes from a flat disk. The high EW tail of the distribution follows a power law with slope of -3.5, which is a natural outcome of such a model. The shape of the distribution below this tail is determined by the intrinsic EW distribution of the sample, which is successfully approximated as the sum of two Gaussian distributions, one centered at $8 \AA$ and one centered at $16 \AA$. It is also shown that the distribution of broad $\mathrm{H} \beta \mathrm{EW}$ is not consistent with a similar model, but suggests that $\mathrm{H} \beta$ is emitted from a flattened surface.

The H08 sample provides new evidence for orientation effects. If the velocity reference system for the 2,265 objects for which [O II] $\lambda 3727$ is measured is changed to that line rather than the [O 


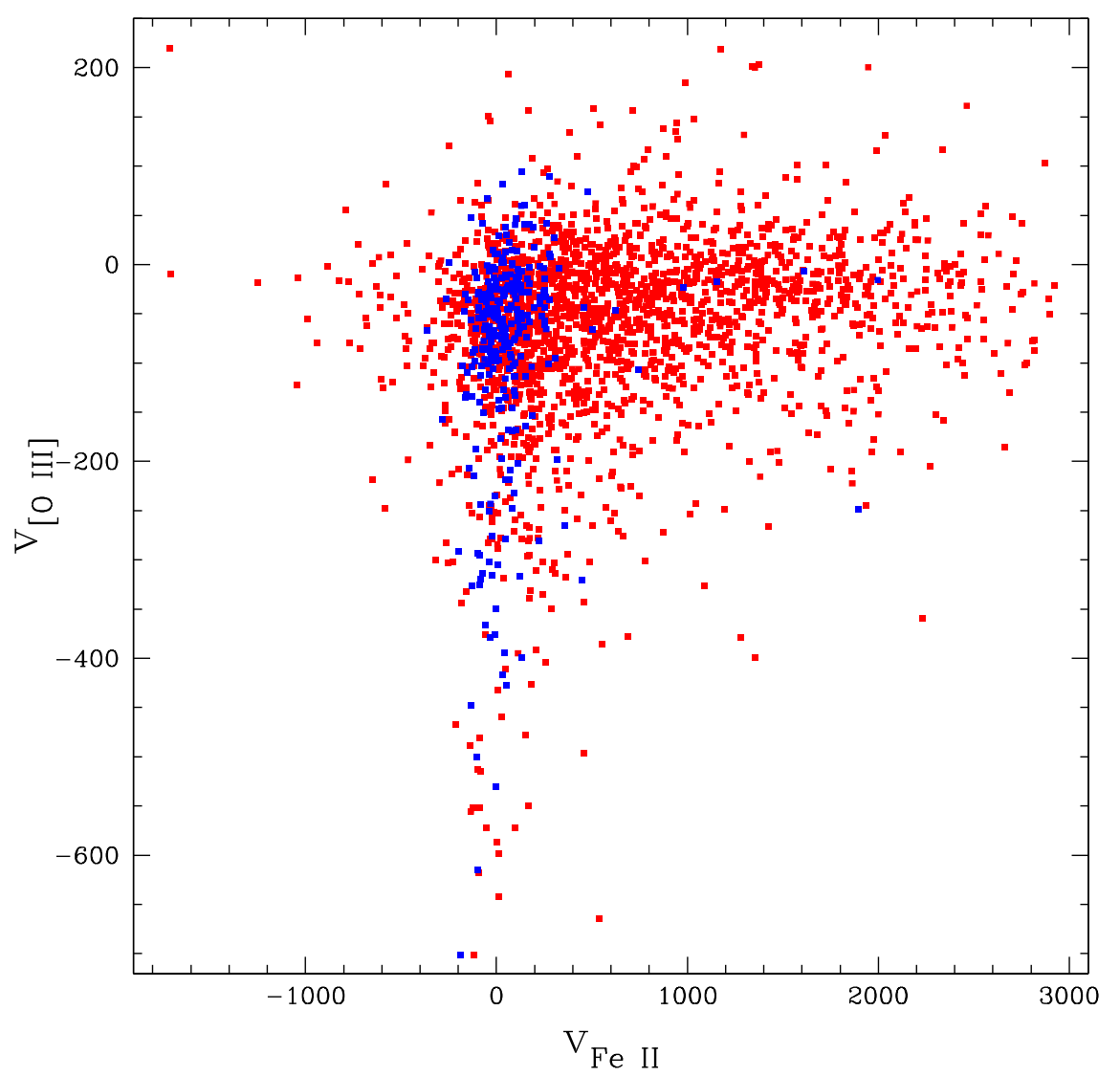

Figure 5: Outflow velocity of [O III] plotted against inflow velocity of Fe II. Both velocities are referenced to [O II[ $\lambda 3727$. NLS1s are shown in blue

III] reference system in the published table, a plot of [O III] relative velocity vs. Fe II relative velocity (Figure 5) shows a notable pattern. Large Fe II inflows only occur in objects with little or no [O III] relative velocity. Large [O III] outflows only occur in objects with little or no Fe II relative velocity. This pattern is indicative of two perpendicular directions of motion, and, in the model described above, suggests that the Fe II inflow is in the plane of the broad-line-emitting disk, while the [O III] outflow is in the polar direction, along the axis of the biconical narrow line region. Thus, objects with large Fe II inflow velocities are predominantly edge-on, and those with large [O III] outflow velocities are predominantly pole-on.

This distinction between edge-on and pole-on objects allows an investigation of whether orientation plays a role in the appearance of NLS1s, and the beginnings of an understanding of the effects of orientation on the observed characteristics of AGNs in general. The first question is answered by Figure 5. The NLS1s, shown in blue, avoid the region populated by edge-on objects. However, they also are not limited to the pole-on region. They dominate that tail, but they are also found in the large clump of objects near the origin, presumably at intermediate orientations. This is not surprising within the context of the model described above. Since there is so little solid angle for objects near pole-on, any sort of realistic intrinsic distribution will result in all bins of the 


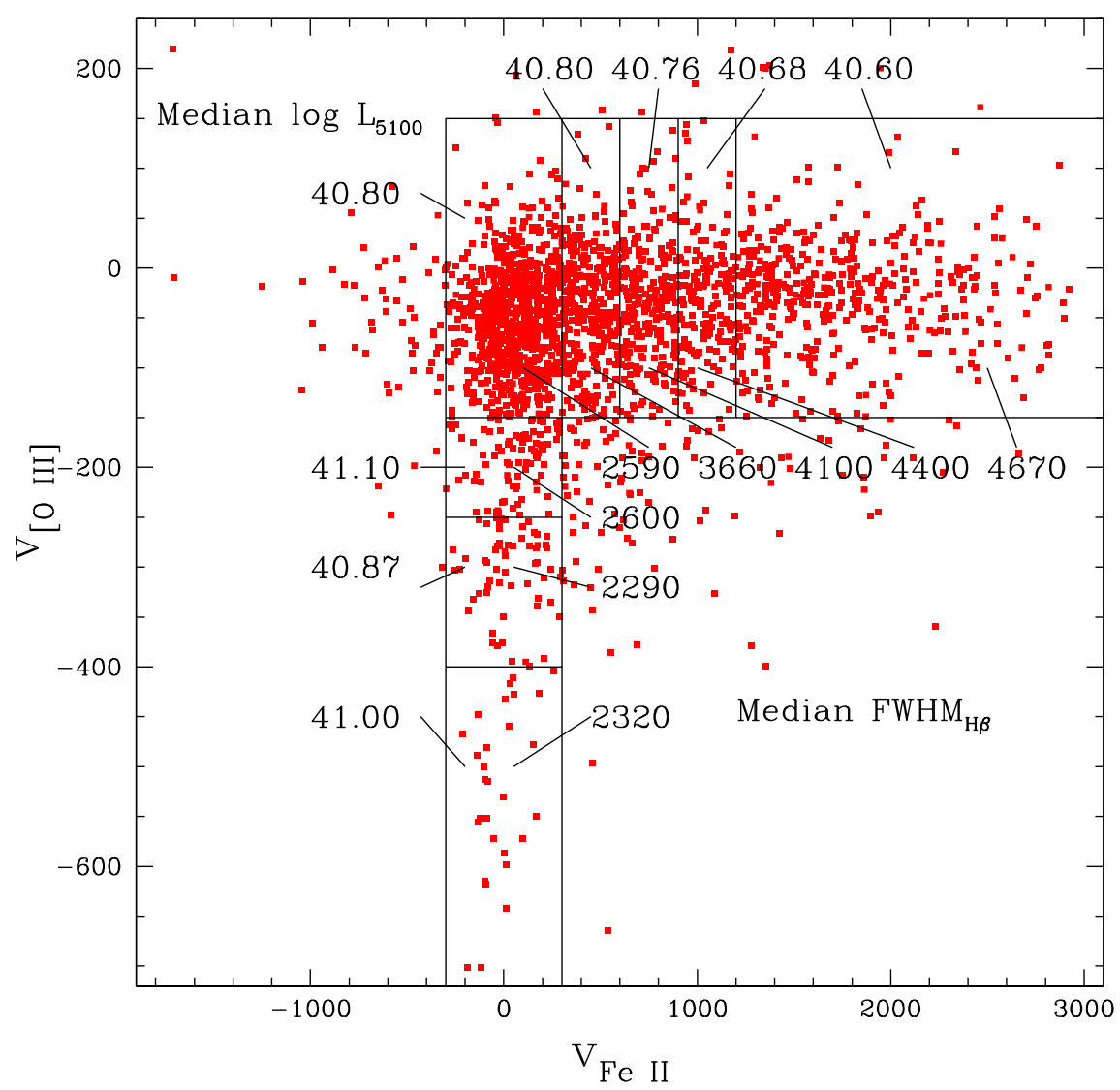

Figure 6: The same data plotted as in figure 5. Four edge-on regions (with Fe II redshift) and three pole-on regions (with [O III] blueshifts) are marked. For each of these, as well as the region around the origin, the median values of $\log \mathrm{L}_{5100}$ and $\mathrm{FWHM}_{\mathrm{H} \beta}$ are indicated.

observed distribution having a significant fraction of non-pole-on objects. This is also consistent with the "edge-on" tail containing a large number of objects, while the "pole-on" tail contains only a few.

Using the Fe II - [O III] velocity diagram, the effects of orientation can be quantified somewhat further. For NLS1s, the characteristics of most interest are luminosity and broad line width, as these define the class and were the properties from which the black hole masses and Eddington ratios were deduced. Figure 6 shows the same diagram of Fe II and [O III] velocities, but here the distribution of points has been divided into eight subsets. The rectangular regions left to right represent subsets with orientations closer and closer to edge-on. The rectangular regions top to bottom represent subsets with orientations closer and closer to pole-on. For each subset, the median values of $\log \mathrm{L}_{5100}$ and $\mathrm{FWHM}_{\mathrm{H} \beta}$ are listed. Just as would be expected, the sequence from edgeon to pole-on corresponds almost monotonically to decreasing broad line width and increasing luminosity. The overall effect is about a factor of two in line width and slightly more than this in luminosity.

Of course, we have not shown that orientation is the only factor that determines an object's 
location in this diagram, and the large clump of objects at the origin suggest that other properties play a role. However, the factor-of-two differences between edge-on and pole-on properties may be indicative of the size of the effect of orientation. With the conclusion that an object seen poleon will be twice as bright and have lines half as wide as the same object seen edge-on, we find that the pole-on object will have an apparent $\mathrm{M}_{\mathrm{BH}}$ roughly one-third of the edge-on object, and an Eddington Ratio almost six times larger. Note that this shift is in the same sense as is seen in Figure 2 - objects with larger $\mathrm{M}_{\mathrm{BH}}$ have smaller Eddington Ratio, but the magnitude of the effect is several times too small to account for the overall trend.

\section{An Integrated Picture?}

Since the discovery of the NLS1 class, one of the fundamental questions surrounding them has been whether they represent a distinct group in which some parameter is "different", or whether they are one end of a continuous distribution of properties. Using large samples available today, weak but highly significant trends can be teased out of the data. Some properties, $\mathrm{EW}_{[\mathrm{OIII}]}$ and $\mathrm{EW}_{\mathrm{FeII}}$, vary smoothly and monotonically with $\mathrm{FWHM}_{\mathrm{H} \beta}$. Other properties, $\mathrm{EW}_{\mathrm{H} \beta}$ and $\mathrm{L}_{5100}$ seem to change behavior dramatically below a certain $\mathrm{FWHM}_{\mathrm{H} \beta}$ value.

It seems clear that orientation, black hole mass, and Eddington ratio all play roles in the apparent characteristics of AGNs. Furthermore, as we begin to disentangle their effects, it appears that these parameters all contribute to the correlations of observed and derived properties comparably. With the acceptance of the virial approach to calculating black hole masses, large complete spectroscopic samples show an inverse correlation between black hole mass and Eddington ratio, implying that NLS1s are the only AGNs that accrete at rates close to their Eddington limits. However, it also seems likely that the two fundamental observables, $\mathrm{FWHM}_{\mathrm{H} \beta}$ and $\mathrm{L}_{5100}$, used to calculate the derived properties, are themselves affected by the orientation of the object relative to our line of sight. The effects of orientation mimic the correlation between $\mathrm{M}_{\mathrm{BH}}$ and Eddington ratio, but are not large enough to be the entire cause of it.

Where does this leave us? With a lot more information, but perhaps not much more insight. Quasars have resisted our attempts for almost 50 years now to create a picture in which observed properties are connected to physical processes in a simple way. As we begin to use large, clearly defined samples of objects to probe the behavior of the observed properties, there is hope that we can (a) separate the intrinsic object-to-object variations from the real trends, and (b) find subtle effects that will allow us to differentiate between physical and geometric parameters.

\section{References}

[1] T. A. Boroson: Blueshifted [O III] Emission: Indications of a Dynamic Narrow-Line Region, AJ 130 (2005) 381 .

[2] T. A. Boroson \& R. F. Green: The Emission-Line Properties of Low-Redshift Quasi-Stellar Objects, ApJS 80 (1992) 109.

[3] R. Decarli et al.:Are the Black Hole Masses in Narrow Line Seyfert 1 Galaxies Actually Small?, MNRAS 386 (2008) L15.

[4] R. Decarli et al.:On the Geometry of Broad Emission Regions in Quasars, MNRAS 387 (2008) 1237. 
[5] C. Hu et al.: A Systematic Analysis of Fe II Emission in Quasars: Evidence for Inflow to the Central Black Hole, ApJ 687 (2008) 78.

[6] S. Komossa: Narrow-Line Seyfert 1 Galaxies, RevMexAA Ser. Conf. 32 (2008) 86.

[7] S. Komossa \& D. Xu:Narrow-Line Seyfert 1 Galaxies and the $M_{\mathrm{BH}^{-}}-\sigma$ Relation, ApJ 667 (2007) L33.

[8] D. E. Osterbrock \& R. W. Pogge: The Spectra of Narrow-Line Seyfert 1 Galaxies, ApJ 297166.

[9] G. Risaliti et al.: [O III] Equivalent Width and Orientation Effects in Quasars, MNRAS 411 (2011) 2223.

[10] D. P. Schneider et al.:The Sloan Digital Sky Survey Quasar Catalog. IV. Fifth Data Release, AJ 134 (2007) 102.

[11] D. P. Schneider et al.:The Sloan Digital Sky Survey Quasar Catalog. V. Seventh Data Release, AJ 139 (2000) 2360.

[12] Y. Shen et al.: A Catalog of Quasar Properties from SDSS DR7, ApJS, in press [arXiv:1006.5178].

[13] C. L. Steinhardt and M. Elvis: The Quasar Mass-Luminosity Plane - I. A Sub-Eddington Limit for Quasars, MNRAS 402 (2010) 2637.

[14] J. W. Sulentic et al.: Eigenvector 1: An Optimal Correlation Space for Active Galactic Nuclei,ApJ 536 (2000) L5.

[15] M.-P. Véron-Cetty et al.: A Spectrophotometric Atlas of Narrow-Line Seyfert 1 Galaxies, A\&A 372 (2001) 730 .

[16] M. Vestergaard and B. M. Peterson: Determining Central Black Hole Masses in Distant Active Galaxies and Quasars. II. Improved Optical and UV Scaling Relations, ApJ 641 (2006) 689.

[17] B. Wills et al.: H Line Width and the UV-X-ray Spectra of Luminous AGN, NewA Rev. 44 (2000) 511.

[18] D. G. York et al.:The Sloan Digital Sky Survey: Technical Summary, AJ 120 (2000) 1579.

[19] R. Zamanov et al.:Kinematic Linkage between the Broad-and Narrow-Line-emitting Gas in Active Galactic Nuclei, ApJ 576 (2002) L9.

[20] H. Zhou et al.: A Comprehenisive Study of 2000 Narrow Line Seyfert 1 Galaxies From the Sloan Digital Sky Survey. I. The Sample, ApJS 166 (2006) 128. 